\title{
Control de asma en adolescentes
}

\author{
Alberto Vidal ${ }^{1}$, Carlos U billa ${ }^{1,2}$, G astón D uffau ${ }^{1}$. \\ Assessment of disease control \\ among asthmatic adolescents
}

Background: The clinical assessment of asthma control is fundamental to evaluate the results of treatment. The Asthma Control Test (ACT) is a questionnaire with five scored items, that differentiates between a well controlled or uncontrolled asthma. It has a Spanish version and has been applied in different countries. Aim: To assess asthma control using the ACT and compare it with the clinical assessment of specialists in a group of pediatric patients. Material and methods: The ACT was applied to 220 adolescents aged 12 to 17 years, 54\% males, with persistent asthma, attended at a respiratory disease unit of a pediatric hospital. The concordance between ACT results and the assessment of specialists was also evaluated. Results: According to ACT and specialists, asthma was controlled in $54 \%$ and $63 \%$ of patients, respectively. There was a weak concordance between ACT and specialists assessment (Kappa Index: 0.27 ; 95\% confidence intervals: $0.14-$ $0.4)$. The degree of asthma control decreased along with increasing severity of the disease $\left(\chi^{2}=\right.$ $10.128, \mathrm{p}=0.001)$. Conclusions: Half of the evaluated asthmatic adolescents do not have an adequate control of their disease. Severity of the disease is inversely related to the degree of control (Rev Méd Chile 2008; 136: 859-66).

(Key words: Adolescent; Asthma; Questionnaires)

\begin{abstract}
Recibido el 12 de noviembre, 2007. Aceptado el 12 de marzo, 2008.
Apoyo financiero: ninguno.

${ }^{1}$ Departamento de Pediatría y Cirugía Infantil, Campus Norte, Facultad de Medicina, Universidad de Chile. ${ }^{2}$ Unidad de Enfermedades Respiratorias del Hospital Roberto del Río. Santiago de Chile.
\end{abstract}

L a medición del grado de control clínico del asma es un pilar fundamental en el tratamiento del paciente asmático. Tradicionalmente se han utilizado muchos métodos para medir el control del asma (CA) en pacientes pediátricos. Entre éstos podemos mencionar el flujo espiratorio máximo, la espirometría, medición de reactividad de vía aérea y más recientemente marcadores de inflamación como óxido nítrico y eosinófilos en esputo ${ }^{1}$.

Correspondencia a: Dr. Alberto Vidal Grell. Holanda 87 Dpto. 604. Providencia, Santiago. Fono celular: 096429701. Fono casa: 4152298. E mail: aevgmd@yahoo.es
La Iniciativa Global para el Asma $^{2}$ hizo un esfuerzo por integrarlos y ha definido el CA en base a siete parámetros, cuatro de éstos centrados en el paciente (síntomas, exacerbaciones, uso de broncodilatador de rescate y limitación de la actividad física), dos que incluyen mediciones fisiológicas (variabilidad del PEF, cercanía al PEF normal) y uno que evalúa los efectos adversos de la terapia. Sin embargo, esta definición no está validada y no incluye marcadores de inflamación.

Existen otros métodos que toman en cuenta la percepción del paciente como cuestionarios de calidad de vida y de control de asma. La Academia Americana de Alergia, Asma e Inmunología (AAAAI en inglés), en conjunto con el Colegio 
Americano de Alergia, Asma e Inmunología (ACA$\mathrm{AI}$, en inglés) publicaron recientemente las recomendaciones para medir el control del asma, basadas en la evidencia científica disponible ${ }^{3}$. Dentro de las recomendaciones se mencionan algunos tests o cuestionarios abreviados de control que han sido validados y publicados ${ }^{4-7}$. Recientemente, Nathan et al publicaron los estudios de validación del Asthma Control Test (ACT), que a través de 5 preguntas con un sistema de puntaje puede diferenciar entre asma bien o mal controlada desde los 12 años, cuenta con una versión en español y ha sido aplicado en países de diferentes continentes ${ }^{8-13}$.

El estudio multinacional "Visión y realidad del asma en Latinoamérica" demostró que sólo 2,4\% de los pacientes logra alcanzar el control de la enfermedad, según lo define la Iniciativa Gobal para el Asma ${ }^{14}$. En nuestro medio sólo encontramos una publicación que muestra los resultados de un programa ordenado de control de adultos asmáticos en una ciudad de la décima región ${ }^{15}$.

Nos interesó conocer el control de la población de adolescentes asmáticos, quienes son un grupo con un espectro clínico variable, frecuentemente subtratados y en que la presencia de factores de riesgo puede llevar al mal control crónico de su asma. Para esto nos propusimos como objetivo principal establecer la prevalencia de control de asma de este grupo a través de un test validado para este fin. Como objetivos secundarios nos planteamos evaluar la concordancia entre el test y la percepción de control del especialista broncopulmonar pediátrico (BCP) y el impacto de la severidad en el control del asma.

\section{MATERIAL Y MÉTOdO}

Durante el período octubre 2006 a julio 2007, se aplicó el ACT, previamente validado y en su versión en español a un grupo de asmáticos adolescentes persistentes, controlados regularmente en la Unidad de Enfermedades Respiratorias del Hospital de Niños Roberto del Río. Se calculó el tamaño muestral necesario para la investigación en base a una muestra piloto con un error aceptable de 5\%. La muestra, que fue configurada al azar, correspondió a 220 asmáticos persistentes entre 12 y 17 años, lo que constituyó aproximadamente el $5 \%$ de los consultantes en el período mencionado.

El ACT tiene 5 preguntas que permiten medir, en las últimas 4 semanas, el grado de control que el paciente asmático le asigna a su enfermedad (Tabla 1). Las respuestas permiten calcular un puntaje total de 5 puntos como mínimo, hasta 25 puntos como máximo. Un puntaje $\geq 20$ puntos corresponde a "asma controlada" y $<20$ puntos a "asma no controlada". El asma controlada se subdivide en "bien controlada", con puntaje de 20 a 24 puntos y "totalmente controlada" con 25 puntos. El asma no controlada en "asma con algún grado de control", con puntaje entre 16 y 19 puntos y "asma pobremente controlada" de 5 a 15 puntos. Los adolescentes respondieron en un lugar privado, sin ayuda y sin límite de tiempo, mientras esperaban su atención médica. Se revisaron las fichas clínicas de los pacientes buscando datos como comorbilidad extrarrespiratoria, tipo de tratamiento y severidad del asma. Además se verificó la percepción de control clínico que el BCP le había asignado a cada paciente en las últimas 4 semanas desde la aplicación del ACT. Hasta el término del estudio, la percepción control realizada por el BCP seguía las recomendaciones de la Iniciativa Global para el Asma ${ }^{2}$. Para el análisis de concordancia entre el ACT y la percepción de control del BCP se utilizó el índice Kappa. El tamaño muestral fue largamente superior al necesario para medir la concordancia. Utilizamos la clasificación de severidad de la Iniciativa Global para el Asma ${ }^{2}$ para dividir la muestra en 3 grupos: persistentes leves, persistentes moderados y persistentes severos. Para comparar el grado de control con la severidad del asma se utilizó $\chi^{2}$ de tendencia $(p<0,05)$. Para el análisis de la comorbilidad nutricional, la muestra fue dividida en tres categorías: eutróficos (IMC entre p10 y <p85), sobrepeso (IMC entre p85 y $<$ p95) y obesos (IMC $\geq$ p95). Se consideró la corrección con estadío de Tanner según la norma nacional de evaluación nutricional para niños entre 6 y 18 años propuesta por el Ministerio de Salud de Chile ${ }^{16}$. Para comparar el grado de control del asma y la patología nutricional por exceso se utilizó $\chi^{2}$ de tendencia $(p<0,05)$. 
Tabla 1. Asma control test

1. En el último mes. ¿Cuánto le ha impedido su asma hacer todo lo que quería

Puntuación en el trabajo, en la escuela o en la casa?

$\begin{array}{ccccc}1 & 2 & 3 & 4 & 5 \\ \text { Siempre } & \text { La mayoría } & \text { Algo del } & \text { Un poco } & \text { Nunca } \\ \text { del tiempo } & \text { tiempo } & \text { del tiempo } & & \end{array}$

2. En el último mes. ¿Con qué frecuencia le ha faltado el aire?

Puntuación

$1 \quad 2 \quad 3 \quad 4$

Más de una

vez por día
Una vez

por día

\section{De 3 a 6 veces} por semana

\section{4}

Una a 2 veces

por semana

3. En el último mes. ¿Con qué frecuencia sus síntomas de asma (respiración sibilante, Puntuación tos, falta de aire, opresión en el pecho o dolor) lo/la despertaron durante la noche o más temprano de lo usual en la mañana?

$\begin{array}{ccccc}1 & 2 & 3 & 4 & 5 \\ 4 \text { o más } & \text { De } 2 \text { a } 3 & \text { Una vez } & \text { Una o dos } & \text { Nunca } \\ \text { noches } & \text { noches } & \text { por semana } & \text { veces } & \\ \text { por semana } & \text { por semana } & & & \end{array}$

4. En el último mes. ¿Con qué frecuencia ha usado su inhalador de rescate o

Puntuación medicamento en nebulizador (como salbutamol)?
1
2
3

3 o más veces Una a dos veces

por día

por día

2 ó 3 veces

por semana

o menos

$\begin{array}{cc}4 & 5 \\ \text { Una vez } & \text { Nunca } \\ \text { por semana } & \end{array}$

5. ¿Cómo evaluaría el control de su asma en el último mes?

Puntuación

1

No controlada

en absoluto
2

Mal

controlada
3

Algo

controlada
4

Bien controlada

\section{Completamente controlada}

Total

Modificado de QualityMetric Incorporated 2002 (Referencia 10).

\section{RESUlTADOS}

Los 220 asmáticos adolescentes incluyeron 102 mujeres $(46,4 \%)$ y $118(53,6 \%)$ hombres. El rango de edad fue de 12 a 17 años, con una mediana de 13 años. La mayoría (80\%) correspondió a pacientes entre 12 y 14 años. Según la severidad del asma se clasificaron como: persistentes leves 30\% (66), persistentes moderados 54,1\% (119) y persistentes severos 15,9\% (35). El 73\% de los asmáticos tenía además rinitis alérgica. Se encontró comorbilidad extrarrespiratoria en $52,3 \%$ de los pacientes. Esta correspondió en su mayoría a obesidad $(23,2 \%)$ y sobrepeso $(22,3 \%)$, seguida de otras 
$(16,4 \%)$ con porcentajes bastante menores al ser consideradas por separado (depresión, talla baja, pubertad precoz o atrasada, hipotiroidismo, déficit atencional, epilepsia, reflujo gastroesofágico y escoliosis leve).

El 95,9\% de la serie se trataba con algún tipo de corticoide inhalado o mezcla de corticoide con beta 2 agonista de acción prolongada.

La Tabla 2 muestra la distribución porcentual del control del asma obtenido mediante ACT y la percepción de control del BCP. Según género, las mujeres tenían un mayor porcentaje de asma controlada que los hombres $(55,9 \%$ y $50,8 \%$, respectivamente) pero sin significado estadístico ( $p=0,455)$. Encontramos 51,1\% de asma controlada en el grupo de 12 a 14 años, mientras que el grupo entre 15 y 17 años, alcanzó $61,4 \%$ de control del asma. Al comparar ambos grupos etarios no encontramos diferencias significativas ( $p=0,223$ ). Según las 4 categorías del ACT, la serie se dividió en: asma totalmente controlada 10,5\%, asma bien controlada $43,2 \%$, asma algo controlada $27,7 \%$ y asma pobremente controlada $18,6 \%$.

Se obtuvo una concordancia débil entre el control obtenido por ACT y la percepción de control del BCP (Tabla 3).

Se encontró asma controlada en $69,7 \%$ de los persistentes leves, $47,1 \%$ de los persistentes moderados y $40 \%$ de los persistentes severos, mostrando una tendencia significativa al mal control mientras mayor fue la severidad de la enfermedad $\left(\chi^{2}\right.$ de tendencia $\left.=10,128 ; p=0,001\right)$.

La patología nutricional por exceso (sobrepeso y obesidad) estaba presente en $45,5 \%$ de los pacientes. Dividimos la serie en asmáticos eutróficos $54,5 \%$, asmáticos en sobrepeso $22,3 \%$ y asmáticos obesos $23,2 \%$. El control de asma se logró en $61,7 \%$ de los eutróficos, $42,9 \%$ de los con sobrepeso y $43,1 \%$ de los obesos, mostrando una tendencia significativa al mal control del asma mientras peor fue la patología nutricional $\left(\chi^{2}\right.$ de tendencia $6,2 ; \mathrm{p}=0,012)$.

Tabla 2. Control de asma en adolescentes mediante ACT y según la percepción del especialista broncopulmonar pediátrico

\begin{tabular}{|lcccccc|}
\hline & $\mathrm{n}$ & $\mathrm{ACT}$ & $\%$ & $\mathrm{n}$ & $\mathrm{BCP}$ & $\%$ \\
\hline Asma controlada & 118 & & 53,7 & 138 & 62,7 \\
Asma no controlada & 102 & & 46,3 & 82 & 37,3 \\
\hline
\end{tabular}

ACT: Asthma Control Test

BCP: Especialista Broncopulmonar Pediátrico

Tabla 3. Concordancia entre control de asma mediante ACT y según la percepción de control del especialista broncopulmonar pediátrico $(\mathrm{n}=\mathbf{2 2 0})$

\begin{tabular}{|clcc|}
\hline & & & ACT \\
& & Controlada & No controlada \\
\hline B & Controlada & 88 & 50 \\
P & No controlada & 29 & 53 \\
\hline
\end{tabular}

Índice Kappa: 0,27 (IC 95\%: 0, 14 - 0,4)

BCP: Especialista Broncopulmonar Pediátrico

ACT: Asma Control Test 


\section{DISCUSIÓN}

Se sabe que un porcentaje importante de los asmáticos no logra controlar su enfermedad, aun bajo la dirección del especialista. Estudios recientes hechos en adultos demuestran que entre $50 \%$ y $55 \%$ de los asmáticos tratados por especialistas no estarían alcanzando el control de la enfermedad ${ }^{17-19}$. En mayores de 12 años, dos publicaciones multinacionales con ACT como instrumento de medición, encontraron $48 \%$ de asma no controlada para Europa y $59 \%$ para $\mathrm{Asia}^{20,21}$.

Nosotros encontramos una prevalencia de asma no controlada ligeramente menor a la reportada en dichos estudios. Sin embargo, nuestra serie tiene un número inferior de pacientes, con un rango etario más estrecho y sólo muestra una realidad local.

Nos preocupa que cerca de la mitad de nuestros pacientes no están alcanzando el control de su enfermedad, pero más preocupante es que $40 \%$ de este grupo corresponde a la subcategoría de pobremente controlados, los que requieren una intervención urgente en el tratamiento.

El estudio original de validación de Nathan demostró una concordancia elevada entre el control obtenido por ACT y el asignado por los especialistas en enfermedades respiratorias. Nosotros encontramos sólo una concordancia débil entre el ACT y el control asignado por el grupo de especialistas. Esto nos hace plantear la siguiente interrogante: ¿Existe una sobreestimación de control del asma por parte del especialista? Históricamente el control del asma ha sido un tema difícil de abordar, tanto por la variabilidad de la enfermedad, como por la incapacidad de encontrar un instrumento adecuado para su medición. Inicialmente se demostró que los pacientes asmáticos tienden a sobreestimar el control de su enfermedad, sin embargo, estudios recientes que comparan los nuevos cuestionarios de control con la opinión de los especialistas, demuestran que existe una ligera tendencia a la sobreestimación por parte de éstos ${ }^{22-25}$. Pensamos que estos instrumentos son un excelente complemento para medir el control clínico del asma, sin embargo no debemos olvidar que es el clínico el que mejor conoce la evolución de la enfermedad en cada paciente, siendo el más capacitado para identificar las variables involucradas en el deterioro del control y realizar las intervenciones necesarias para mejorarlo.

Un tema recurrente en los últimos años es la relación entre severidad y control del asma. Se acepta que son dos conceptos diferentes, pero que tienen parámetros de evaluación que se sobreponen con frecuencia. La severidad del asma define la gravedad intrínseca de la enfermedad, siendo más útil en el diagnóstico, como enfrentamiento inicial y sin uso de terapia. El concepto de control describe el estado clínico del asma, que es variable en el tiempo, permitiendo el seguimiento del paciente, mediante la respuesta clínica a la terapia ${ }^{26-28}$. Es frecuente que los asmáticos leves y moderados tengan un mejor control que los severos. Esto lo demostramos al encontrar peor control de la enfermedad, mientras mayor es la severidad del asma y confirma la superposición de algunos parámetros clínicos de ambos conceptos. Sin embargo, nuestros porcentajes de asmáticos severos y asmáticos no controlados, difieren sustancialmente $(15,9 \%$ y $43,7 \%$, respectivamente), respaldando el hecho de que asma severa y asma no controlada no son sinónimos.

La literatura describe algunos alergenos inhalatorios y contaminantes ambientales (ácaros, hongos, tabaquismo, ozono), como factores de mal control de asma, potencialmente modificables ${ }^{29-35}$. Otros, se relacionan directamente con el paciente como la adherencia al tratamiento, técnica inhalatoria, estrés emocional y uso de fármacos contraindicados en la enfermedad ${ }^{36-38}$. La rinitis alérgica es una patología muy frecuente en asma, hecho que corroboramos en nuestro estudio. La evidencia demuestra que el adecuado tratamiento de la rinitis mejora el control del asma, por lo que grupos de expertos recomiendan un plan de tratamiento en conjunto ${ }^{39-41}$.

Aunque no fue uno de los objetivos iniciales del estudio evaluar el impacto de la comorbilidad en el control del asma, destacamos que más de la mitad de la serie tenía algún tipo de comorbilidad no respiratoria. La mayoría correspondió a sobrepeso y obesidad, patologías relacionadas con mayor incidencia, mayor frecuencia de síntomas e incremento de hospitalizaciones por asma en pacientes pediátricos ${ }^{42-44}$. Además, logramos verificar que el control del asma empeora a mayor severidad de la patología nutricional, lo que había sido reportado previamente en asmáticos adultos ${ }^{45-47}$. 
Pensamos que futuras investigaciones deberían incentivar el conocimiento de los factores de mal control con mayor importancia en nuestra realidad. Esto permitiría diseñar algoritmos de manejo con enfoque individual y optimizar el tratamiento. Otro punto importante a investigar es el control de asma en escolares, quienes en nuestro medio tienen una mayor prevalencia de asma que los adolescentes y probablemente diferencias en cuanto a los factores de mal control de asma $^{48}$. Para este grupo, especialmente en los menores de 12 años, ya se cuenta con un test abreviado de control de asma con preguntas dirigidas a ellos y a sus padres ${ }^{49}$.

Estamos convencidos que el control del asma es un concepto amplio e integrador que debe ser interpretado adecuadamente por el especialista,

\section{REFERENCIAS}

1. Deykin A. Biomarker-driven care in asthma: Are we there? J Allergy Clin Immunol 2006; 118: 565-8.

2. Global Initiative for Asthma, Global Strategy for Asthma Management and Prevention. Management Segment, Chapter 7; NIH Publication № 02-3659; issued January 1995 (updated 2002), updated 2005 from the 2004 document. Available at: http:// www.ginasthma.org [Consultado el 15 de agosto de 2006].

3. L J J, Oppenheimer J, Bernstein L, Nickias R. Attaining optimal asthma control: A practice parameter. J Allergy Clin Immunol 2005; 116: S3-S11.

4. Juniper E, O’Byrne P, Guyatt G, Ferrie P, King D. Development and validation of a questionnaire to measure asthma control. Eur Respir J 1999; 14: 902-7.

5. Volmer W, Markson L, O'CONNOR E, SANOCKI L, FitTerman L, Berger M et al. Association of asthma control with health care utilization and quality of life. Am J Respir Crit Care Med 1999; 160: 1647-52.

6. Volumer W, Markson L, O'Connor E, Frazier E, Berger M, BuIST A. Association of asthma control with health care utilization: a prospective evaluation. Am J Respir Crit Care Med 2002; 165: 195-9.

7. Skinner E, Diette G, Algatt-Bergstrom P, Nguyen T, Ciark R, Markson L et al. The Asthma Therapy Assessment Questionnaire (ATAQ) for Children and Adolescents. Disease Management 2004; 7: 305-13.

8. Nathan R, Sorkness C, Kosinski M, Schatz M, Li J, MARCus P ET AL. Development of the asthma control test: a survey for assessing asthma control. J Allergy Clin Immunol 2004; 113: 59-65. teniendo en cuenta las múltiples variables clínicas implicadas y también la percepción del paciente, por lo que la medición del control a través del ACT puede ser una variable más que complemente la evaluación global.

Finalmente, podemos concluir que los adolescentes asmáticos de la Unidad de Enfermedades Respiratorias del Hospital Roberto del Río tienen una prevalencia elevada de mal control de su enfermedad, que no difiere de lo reportado en publicaciones internacionales. El control del asma obtenido con ACT tiene una débil concordancia con la percepción de control del especialista, por lo que estos resultados deben ser interpretados con cautela, considerando la revisión de los criterios clínicos de control de asma que utilizamos en los pacientes.

9. Schatz M, Sorkness C, L J, Marcus P, Murray J, Nathan RA ET AL. Asthma control test: Reliability, validity, and responsiveness in patients not previously followed by asthma specialists. J Allergy Clin Immunol 2006; 117: 549-56.

10. GlaxoSmithKline, Inc; QualityMetrics, Inc. Copyright 2005. Asthma Control Test. Available at: http:// www.asthmacontrol.com/index_es.html [Consultado el 10 de agosto de 2006].

11. Xin Z, Feng-Ming D, Jiang-Tao L, Kai-Sheng Y, Ping C, QuAN-Y ING H ET AL. Validity Of Asthma Control Test In Chinese Patients. Chin Med J (Engl) 2007; 120: 1037-41.

12. Ahmed S, Ernst P, Tamblyn R, Colman N. Validation of The 30 Second Asthma Test ${ }^{\mathrm{TM}}$ as a measure of asthma control. Can Resp J 2007; 14: 105-9.

13. Laforest L, Van Ganse E, Devouassoux G, Bousquet J, Chretin S, Bauguil G eT al. Influence of patients' characteristics and disease management on asthma control. J Allergy Clin Immunol 2006; 117: 1404-10.

14. Nefren H, Fritscher C, Cuevas F, Levy G, Chiarelia P, Soriano J, Mechali D. Asthma control in Latin America: the Asthma Insights and Reality in Latin America (AIRLA) survey. Rev Panam Salud Publica 2005; 17: 191-7.

15. Riquelme M, Riquelme R, Martínez D. Experiencia de un Programa de Control de Asma Bronquial en Puerto Montt, Chile. Rev Chil Enf Respir 2006; 22: 93-7.

16. Pizarro T, Rodríguez L, Benavides X. Norma Técnica de Evaluación Nutricional del Niño de 6 a 18 años. Año 2003. Rev Chil. Nutr 2004; 31: 128-37. 
17. Gaga M, Papageorgiou N, Zervas E, Gioulekas D, Konstantopoulos S. Control of Asthma Under Specialist Care Is It Achieved? Chest 2005; 128: 78-84.

18. Peters S, Jones C, Haselkorn T, Mink D, Valacer D, WeIss S. Real-world Evaluation of Asthma Control and Treatment (REACT): findings from a national Web-based survey. J Allergy Clin Immunol 2007; 119: 1454-61.

19. LeNoir M, Wiluamson A, Stanford R, Stempel D. Assessment of asthma control in a general population of asthmatics Curr Med Res Opin 2006; 22: 17-22.

20. Vervloet D, Wiliams A, Lloyd A, Clark T. Costs of managing asthma as defined by a derived Asthma Control TestTM score in seven European countries. Eur Respir Rev 2006; 15: 17-23.

21. Lai C, Kuo S, De Guia T, Lloyd A, Wiluams A, Spencer M. Asthma control and its direct healthcare costs: findings using a derived Asthma Control TestTM score in eight Asia-Pacific Areas. Eur Respir Rev 2006; 15: 24-9.

22. Boulet LP, Phiwips R, O’Byrne P, Becker A. Evaluation of asthma control by physicians and patients: comparison with current guidelines. Can Respir J 2002; 9: 417-23.

23. Juniper E, Chauhan A, Nevilie E, Chatterjee A, Svensson K, MöRK A ET AL. Clinicians tend to overestimate improvements in asthma control: an unexpected observation. Prim Care Respir J 2004; 13: 181-4.

24. Juniper E, Bousquet J, Abetz L, Bateman E. Identifying 'well-controlled' and 'not well-controlled' asthma using the Asthma Control Questionnaire. Respir Med 2006; 100: 616-21.

25. JuniPER E. Assessing Asthma Control. Curr Allergy Asthma Rep 2007; 7: 390-4.

26. Funlbrigge A. Asthma severity and asthma control: symptoms, pulmonary function, and inflammatory markers. Curr Opin Pulm Med 2004; 10: 1-6.

27. Yawn B, Brenneman S, Auen-Ramey F, Cabana M, MARKSON L. Assessment of Asthma Severity and Asthma Control in Children. Pediatrics 2006; 118: 322-9.

28. Humbert M, Holgate S, Boulet L, Bousquet J. Asthma control or severity: that is the question. Allergy 2007; 62: 95-101.

29. Morgan W, Crain E, Gruchalla R, O’Connor G, Kattan M, Evans R ET AL. Results of a Home-Based Environmental Intervention among Urban Children with Asthma. N Engl J Med 2004; 351: 1068-80.

30. Halken S, Host A, Nikiassen U, Hansen LG, Nielsen F, Pedersen S ET AL. Effect of mattress and pillow encasings on children with asthma and house dust mite allergy. J Allergy Clin Immunol 2003; 111: 169-76.

31. Denning D, O’Driscoll B, Hogaboam C, Bowyer P, NIvEN R. The link between fungi and severe asthma: a summary of the evidence. Eur Respir J 2006; 27: 615-26.

32. Chaudhuri R, Livingston E, McMahon A, Thomson L, Borland W, THOMSON NC. Cigarette smoking impairs the therapeutic response to oral corticosteroids in chronic asthma. Am J Respir Crit Care Med 2003; 168: 1308-11.

33. Chalmers G, Macleod K, Little S, Thomson L, McSharry C, THOMson N. Influence of cigarette smoking on inhaled corticosteroid treatment in mild asthma. Thorax 2002; 57: 226-30.

34. Dales R, Cakmak S, Judek S, Dann T, Coates F, Brook JR ET AL. Influence of outdoor aeroallergens on hospitalization for asthma in Canada. J Allergy Clin Immunol 2004; 113: 303-6.

35. Chen L, Tager I, Peden D, Christian D, Ferrando R, WELCH B ET AL. Effect of ozone exposure on airway responses to inhaled allergen in asthmatic subjects. Chest 2004; 125: 2328-35.

36. Szczekuk A, Nizankowska E, Bochenek G, Nagraba K, Mejza F, SwierczynSKa M. Safety of a specific COX-2 inhibitor in aspirin-induced asthma. Clin Exp Allergy 2001; 31: 219-25.

37. Covar R, Macomber B, Szefler S. Medications as asthma triggers. Immunol Allergy Clin North Am 2005; 25: 169-90.

38. Sandberg S, Paton J, Ahola S, McCann D, McGuinness D, Hillary C ET AL. The role of acute and chronic stress in asthma attacks in children. Lancet 2000; 356: 982-7.

39. Bousouet J, Van Caumenberge P, Khaltaev N. Allergic rhinitis and its impact on asthma. J Allergy Clin Immunol 2001; 108: 147-334.

40. Thomas M. Allergic rhinitis: evidence for impact on asthma. BMC Pulm Med 2006; 6: S4.

41. Adams R, Fuhlbrigge A, Finkelstein J, Weiss S. Intranasal steroids and the risk of emergency department visits for asthma. J Allergy Clin Immunol 2002; 109: 636-42.

42. SHORE S. Obesity and asthma: implications for treatment. Curr Opin Pulm Med 2007; 13: 56-62.

43. Castro-Rodríguez J, Holberg C, Morgan W, Wright A, MARTínEZ F. Increased Incidence of Asthmalike Symptoms in Girls Who Become Overweight or Obese during the School Years. Am J Respir Crit Care Med 2001; 163: 1344-9.

44. Carroll C, Stoltz P, Raykov N, Smith S, Zucker A. Childhood Overweight Increases Hospital Admission Rates for Asthma Aaron R. Pediatrics 2007; 120: 734-40.

45. Lavoie K, Bacon S, Labrecque M, Cartier A, Ditto B. Higher BMI is associated with worse asthma control and quality of life but not asthma severity. Respir Med 2006; 100: 648-57.

46. Stenius-Aarniala B, Poussa T, Kvarnström J, Grönlund E, Ylikahri M, Mustajoki P. Immediate and long term 
effects of weight reduction in obese people with asthma: randomized controlled study. BMJ 2000; 320: 827-32.

47. Saint-Pierre P, Bourdin A, Chanez P, Daures J, Godard P. Are overweight asthmatics more difficult to control? Allergy 2006: 61: 79-84.

48. Malol J, Cortez E, Amarales L, Sánchez I, Calvo M, Soto $\mathrm{S}$ ET AL. Prevalencia del asma en escolares chilenos:
Estudio descriptivo de 24.470 niños. ISAAC-Chile. Rev Méd Chile 2000; 128: 279-85.

49. Liu A, Zeiger R, Sorkness C, Mahr T, Ostrom N, Burgess S ET AL. Development and cross-sectional validation of the Childhood Asthma Control Test. J Allergy Clin Immunol 2007; 119: 817-25. 